\title{
On an Invariant Borel Measure in Hilbert Space
}

by

\author{
G. PANTSULAIA
}

Presented by Andrzej LASOTA

Summary. An example of a nonzero $\sigma$-finite Borel measure $\mu$ with everywhere dense linear manifold $\mathbb{I}_{\mu}$ of admissible (in the sense of invariance) translation vectors is constructed in the Hilbert space $\ell_{2}$ such that $\mu$ and any shift $\mu^{(a)}$ of $\mu$ by a vector $a \in \ell_{2} \backslash \mathbb{I}_{\mu}$ are neither equivalent nor orthogonal. This extends a result established in [7].

We first recall some standard notions from measure theory. Let $E$ be a complete metric linear space, by which we mean a vector space with a complete metric for which addition and scalar multiplication are continuous. Let $B(E)$ be the $\sigma$-algebra of Borel subsets of $E$, and $\mu$ be a nonzero nonnegative measure defined on $B(E)$. We write $X+a$ for the translation of a set $X \subseteq E$ by a vector $a \in E$. The measure $\mu^{(a)}$ defined by $\mu^{(a)}(X)=\mu(X-a)$ for $X \in B(E)$ is called the shift of the measure $\mu$ by $a$.

Definition 1. A vector $a \in E$ is called an admissible translation vector for $\mu$ in the sense of quasiinvariance if the measures $\mu$ and $\mu^{(a)}$ are equivalent. We denote the set of all such vectors by $\mathbb{Q}_{\mu}$.

Definition 2. A vector $a \in E$ is called an admissible translation vector for $\mu$ in the sense of invariance if $\mu=\mu^{(a)}$. We denote by $\mathbb{I}_{\mu}$ the set of all such vectors.

REMARK 1. It is obvious that $\mathbb{I}_{\mu} \subseteq \mathbb{Q}_{\mu}$ for every $\mu$. One can easily show that in the case of Lebesgue measure the above-mentioned sets coincide. If we consider the canonical Gaussian measure $\gamma$ on the topological vector space $\mathbb{R}^{\mathbb{N}}$, then according to Kakutani's [10] well known result the set $\mathbb{Q}_{\gamma}$ coincides with $\ell_{2}$, while $\mathbb{I}_{\gamma}$ consists only of the zero vector.

2000 Mathematics Subject Classification: 28A35, 28C20, $60 \mathrm{G} 15$.

Key words and phrases: admissible translation, Gaussian measure, quasiinvariance, invariance. 
Among the main works devoted to quasiinvariance of measures in general linear spaces one should mention especially the classical work of S. Kakutani [10], where equivalence and orthogonality of infinite product measures are investigated. It is shown that if one has equivalent probability measure $\mu_{i}$ and $\nu_{i}$ on a $\sigma$-algebra $\mathcal{L}_{i}$ of subsets of a set $\Omega_{i}, i=1,2, \ldots$, and if $\mu$ and $\nu$ denote respectively the infinite product measures $\prod_{i \in \mathbb{N}} \mu_{i}$ and $\prod_{i \in \mathbb{N}} \nu_{i}$ on the infinite product $\sigma$-algebra generated on $\Omega=\prod_{i \in \mathbb{N}} \Omega_{i}$, then $\mu$ and $\nu$ are either equivalent or orthogonal. Similar dichotomies have revealed themselves in the study of Gaussian stochastic processes. C. Cameron and W. E. Martin proved in [4] that if one considers the measures induced on the path space by two Wiener processes on the unit interval, and the variances of the processes are different, then the measures are orthogonal. Results of this sort were generalized by many authors (cf. [2], [3], [6], [8], [13], [15] and others). Their techniques allow us to construct Gaussian measures in general linear spaces with everywhere dense linear manifolds of admissible translation vectors in the sense of quasiinvariance. In the context of the above-mentioned results, I. I. Gikhman and A. V. Skorokhod [7, p. 556] considered the following

Problem. Does there exist a probability Borel measure $\mu$ in $\ell_{2}$ which satisfies the following conditions:

(i) the group $\mathbb{Q}_{\mu}$ is an everywhere dense linear manifold in $\ell_{2}$,

(ii) there exists $a \in \ell_{2} \backslash \mathbb{Q}_{\mu}$ such that $\mu$ is not orthogonal to $\mu^{(a)}$ ?

Their solution depended on the technique of Gaussian measures in infinitedimensional separable Hilbert space.

The purpose of the present paper is to extend Gikhman-Skorokhod's above-mentioned result to invariant Borel measures in $\ell_{2}$.

We will construct nonzero $\sigma$-finite Borel measures in $\mathbb{R}^{\mathbb{N}}$ which are invariant with respect to everywhere dense linear manifolds.

Let $\mathbb{R}^{\mathbb{N}}$ be the space of all sequences of real numbers equipped with the Tikhonov topology. Denote by $B\left(\mathbb{R}^{\mathbb{N}}\right)$ the $\sigma$-algebra of all Borel subsets in $\mathbb{R}^{\mathbb{N}}$. Let $\left(a_{i}\right)_{i \in \mathbb{N}}$ and $\left(b_{i}\right)_{i \in \mathbb{N}}$ be sequences of real numbers such that $a_{i}<b_{i}$ for every $i \in \mathbb{N}$. Put

$$
A_{n}=\mathbb{R}_{0} \times \ldots \times \mathbb{R}_{n} \times\left(\prod_{i>n} \Delta_{i}\right) \quad(n \in \mathbb{N}),
$$

where

$$
\mathbb{R}_{i}=\mathbb{R}, \quad \Delta_{i}=\left[a_{i}, b_{i}[, \quad \forall i \in \mathbb{N} .\right.
$$

For $i \in \mathbb{N}$, consider the Lebesgue measure $\mu_{i}$ on $\mathbb{R}_{i}$ with $\mu_{i}\left(\Delta_{i}\right)=1$. Denote by $\lambda_{i}$ the normalized Lebesgue measure defined on $\Delta_{i}$. 
For $n \in \mathbb{N}$, denote by $\nu_{n}$ the measure defined by

$$
\nu_{n}=\prod_{1 \leq i \leq n} \mu_{i} \times \prod_{i>n} \lambda_{i}
$$

and by $\bar{\nu}_{n}$ the Borel measure in $\mathbb{R}^{\mathbb{N}}$ defined by

$$
\bar{\nu}_{n}(X)=\nu_{n}\left(X \cap A_{n}\right) \quad \text { for } X \in B\left(\mathbb{R}^{\mathbb{N}}\right) .
$$

We need the following lemmas, proved in [12]:

Lemma 1. For every Borel set $X \subseteq \mathbb{R}^{\mathbb{N}}$ the limit

$$
\nu_{\Delta}(X)=\lim _{n \rightarrow \infty} \bar{\nu}_{n}(X)
$$

exists. Moreover, the functional $\nu_{\Delta}$ is a nontrivial $\sigma$-finite measure on $B\left(\mathbb{R}^{\mathbb{N}}\right)$.

LEMMA 2. The set $\mathbb{I}_{\mu_{\Delta}}$ consists of all $g=\left(g_{1}, g_{2}, \ldots\right)$ for which the series $\sum_{i=1}^{\infty}\left|g_{i}\right| /\left(b_{i}-a_{i}\right)$ converges.

REMARK 2. The construction of the measure $\nu_{\Delta}$ belongs to A. B. Kharazishvili [11].

The main result can now formulated as follows.

THEOREM. There exists a nonzero $\sigma$-finite Borel measure $\mu$ in $\ell_{2}$ which satisfies the following conditions:

(i) The group $\mathbb{I}_{\mu}$ is an everywhere dense linear manifold in $\ell_{2}$.

(ii) There exists $a \in \ell_{2} \backslash \mathbb{I}_{\mu}$ such that the measure $\mu^{(a)}$ is not orthogonal to $\mu$.

Proof. Clearly,

$$
\ell_{2}=\bigcup_{n \in \mathbb{N}} \bigcap_{m \in \mathbb{N}}\left\{\left(x_{k}\right)_{k \in \mathbb{N}}: \sum_{k=1}^{m} x_{k}^{2} \leq n\right\} .
$$

Since $\left\{\left(x_{k}\right)_{k \in \mathbb{N}}: \sum_{k=1}^{m} x_{k}^{2} \leq n\right\} \in B\left(\mathbb{R}^{\mathbb{N}}\right)$ for $m, n \in \mathbb{N}$, we conclude that $\ell_{2} \in B\left(\mathbb{R}^{\mathbb{N}}\right)$. Hence, $B\left(\ell_{2}\right) \subseteq B\left(\mathbb{R}^{\mathbb{N}}\right)$. For $X \in B\left(\ell_{2}\right)$, we put

$$
\mu_{1}(X)=\nu_{\Delta_{1}}(X), \quad \mu_{2}(X)=\nu_{\Delta_{2}}(X),
$$

where

$$
\Delta_{1}=\prod_{i \in \mathbb{N}}\left[0, \frac{1}{i+1}\left[, \quad \Delta_{2}=\prod_{i \in \mathbb{N}}\left[0, \frac{1}{(i+1) 2^{i}}[.\right.\right.\right.
$$

According to Lemma 2, we have

$$
\begin{aligned}
& \mathbb{I}_{\mu_{1}}=\left\{\left(g_{i}\right)_{i \in \mathbb{N}} \in \ell_{2}: \sum_{i \in \mathbb{N}}\left|g_{i}\right|(i+1)<\infty\right\}, \\
& \mathbb{I}_{\mu_{2}}=\left\{\left(h_{i}\right)_{i \in \mathbb{N}} \in \ell_{2}: \sum_{i \in \mathbb{N}}\left|h_{i}\right| 2^{i}(i+1)<\infty\right\} .
\end{aligned}
$$


It is clear that $\mathbb{I}_{\mu_{2}} \subset \mathbb{I}_{\mu_{1}}$. Morover, the measures $\mu_{1}$ and $\mu_{2}$ are orthogonal. Indeed, $\mu_{2}$ is concentrated on the set

$$
F_{2}=\bigcup_{n \in \mathbb{N}} \mathbb{R}^{n} \times \prod_{i>n}\left[0, \frac{1}{(i+1) 2^{i}}[\right.
$$

and $\mu_{1}\left(F_{2}\right)=0$.

We set $\mu=\mu_{1}+\mu_{2}$. Note that the group $\mathbb{I}_{\mu}$ coincides with $\mathbb{I}_{\mu_{2}}$. It is also clear that $\mathbb{I}_{\mu}$ is a linear manifold. The fact that $\mathbb{I}_{\mu}$ is everywhere dense in $\ell_{2}$ is a simple consequence of the fact that the linear manifold of all sequences with a finite number of nonzero terms is everywhere dense in $\ell_{2}$. Consider

$$
a=\left(\frac{1}{(1+i) 2^{i}}\right)_{i \in \mathbb{N}} \in \mathbb{I}_{\mu_{1}} \backslash \mathbb{I}_{\mu_{2}} .
$$

Then the measure $\mu$ is not orthogonal to $\mu^{(a)}$, because $\mu^{(a)}$ has an absolutely continuous component $\mu_{2}^{(a)}$ with respect to $\mu$.

REMARK 3. If we consider probability measures $\lambda_{1}$ and $\lambda_{2}$ which are equivalent to the measures $\mu_{1}$ and $\mu_{2}$, respectively, then the measure $\lambda=$ $\frac{1}{2}\left(\lambda_{1}+\lambda_{2}\right)$ is a solution of the above-mentioned problem. Hence, the Theorem may be regarded as a generalization of the result obtained in [7].

\section{References}

[1] V. V. Baklan and A. D. Shatashvili, Transformations of Gaussian measures by non-linear mappings in Hilbert space, Dopovīdī Akad. Nauk Ukraïn. RSR 1965, $1115-1117$.

[2] R. H. Cameron and W. T. Martin, Transformations of Wiener integrals under translations, Ann. of Math. (2) 45 (1944), 386-396.

[3] -, - , Transformations of Wiener integrals under a general class of linear transformations, Trans. Amer. Math. Soc. 58 (1945), 184-219.

[4] - - - The behavior of measure and measurability under change of scale in Wiener space, Bull. Amer. Math. Soc. 53 (1947), 130-137.

[5] - - - The transformation of Wiener integrals by nonlinear transformations, Trans. Amer. Math. Soc. 66 (1949), 253-283.

[6] J. Feldman, Equivalence and perpendicularity of Gaussian processes, Pacific J. Math. 8 (1958), 699-708.

[7] I. I. Gikhman and A. V. Skorokhod, Theory of Random Processes, Vol. I, Izdat. Nauka, Moscow, 1971 (in Russian).

[8] U. Grenander, Stochastic processes and statistical inference, Ark. Mat. 1 (1950), 195-277.

[9] P. R. Halmos, Measure Theory, Van Nostrand, New York, 1950.

[10] S. Kakutani, On equivalence of infinite product measures, Ann. of Math. 49 (1948), 214-224.

[11] A. B. Kharazishvili, Invariant measures in Hilbert space, Soobshch. Akad. Nauk Gruzin. SSR 114 (1984), 45-48 (in Russian). 
[12] G. Pantsulaia, Duality of measure and category in infinite-dimensional separable Hilbert space $l_{2}$, Int. J. Math. Math. Sci. 30 (2002), 353-363.

[13] T. S. Pitcher, Likelihood ratios for diffusion processes with shifted mean values, Trans. Amer. Math. Soc. 101 (1961), 168-176.

[14] V. N. Sudakov, Linear sets with quasi-invariant measure, Dokl. Akad. Nauk SSSR 127 (1959), 524-525 (in Russian).

[15] A. M. Vershik, Duality in the theory of measure in linear spaces, ibid. 170 (1966), 497-500 (in Russian).

G. Pantsulaia

Department of Mathematics

Georgian Technical University

77 Kostava St.

0175 Tbilisi 75, Georgia

E-mail: gogi@mail.gtu.edu.ge 